\title{
New Insights into the Structural Roles of Nebulin in Skeletal Muscle
}

\author{
Coen A. C. Ottenheijm ${ }^{1,2}$ and Henk Granzier ${ }^{2}$ \\ ${ }^{1}$ Laboratory for Physiology, Institute for Cardiovascular Research, VU University Medical Center, \\ 1081 BT Amsterdam, The Netherlands \\ ${ }^{2}$ Department of Physiology, University of Arizona, P.O. Box 245217, Tucson, AZ 85724, USA
}

Correspondence should be addressed to Henk Granzier, granzier@email.arizona.edu

Received 11 January 2010; Accepted 26 March 2010

Academic Editor: Guy M. Benian

Copyright ( $) 2010$ C. A. C. Ottenheijm and H. Granzier. This is an open access article distributed under the Creative Commons Attribution License, which permits unrestricted use, distribution, and reproduction in any medium, provided the original work is properly cited.

\begin{abstract}
One important feature of muscle structure and function that has remained relatively obscure is the mechanism that regulates thin filament length. Filament length is an important aspect of muscle function as force production is proportional to the amount of overlap between thick and thin filaments. Recent advances, due in part to the generation of nebulin KO models, reveal that nebulin plays an important role in the regulation of thin filament length. Another structural feature of skeletal muscle that is not well understood is the mechanism involved in maintaining the regular lateral alignment of adjacent sarcomeres, that is, myofibrillar connectivity. Recent studies indicate that nebulin is part of a protein complex that mechanically links adjacent myofibrils. Thus, novel structural roles of nebulin in skeletal muscle involve the regulation of thin filament length and maintaining myofibrillar connectivity. When these functions of nebulin are absent, muscle weakness ensues, as is the case in patients with nemaline myopathy with mutations in nebulin. Here we review these new insights in the role of nebulin in skeletal muscle structure.
\end{abstract}

\section{Nebulin Specifies Thin Filament Length}

Skeletal muscles are organized into regular arrays of thin and thick filaments of well-defined length. Filament length is an important aspect of muscle function because a muscle generates force in proportion to thin and thick filament overlap. Whereas thick filament length is considered a constant $1.6 \mu \mathrm{m}$, thin filament lengths are fine-tuned at $\sim 1.0$ $1.3 \mu \mathrm{m}$, depending on species and muscle type [1] to overlap with thick filaments and to meet the muscle's physiological demands $[1,2]$. Changes in thin filament length affect thin-thick filament overlap and impact a muscle's forcegenerating capacity at a given sarcomere length: thus, thin filament length is a key aspect of muscle function. Since length is not an intrinsic property of actin-filaments (actin monomers assemble in vitro to highly variable polymer lengths [3]), thin filament length is likely to be specified in vivo by an actin binding protein; for this, nebulin has been considered for a long time a prime candidate [4-6], but critical evidence has been lacking up until recently.
Nebulin is a giant protein $(\mathrm{Mw} 700-800 \mathrm{kDa})$ expressed in skeletal muscle, and makes up $2 \%-3 \%$ of the myofibrillar protein mass. Immuno-electronmicroscopy revealed that a single nebulin molecule spans the thin filament with its $\mathrm{C}$-terminus anchored at the $\mathrm{Z}$-disk and its $\mathrm{N}$-terminal region directed towards the thin filament pointed end (for a schematic representation, see Figure 1(a)) [4]. The first evidence for nebulin's proposed role in specifying thin filament length came from the analysis of nebulin's cDNA sequence. This revealed that the bulk of the molecule is comprised of modules with the centrally located modules M9 to M162, each thought to represent individual actin-binding motifs and organized into seven-module superrepeats that match the repeat of the actin filament (Figure 1(b)). This precise arrangement is thought to allow each nebulin module to interact with a single monomer of the actin filament $[5,6]$, and each nebulin super-repeat to associate with a single tropomyosin ( $\mathrm{Tm}$ )/troponin ( $\mathrm{Tn}$ ) complex [7-9]. Nebulin's extreme N-terminal modules M1-M3 (Figure 1(b)) contain a high-affinity binding site for the thin filament pointedend capping protein tropomodulin [10]. Tropomodulin, in 


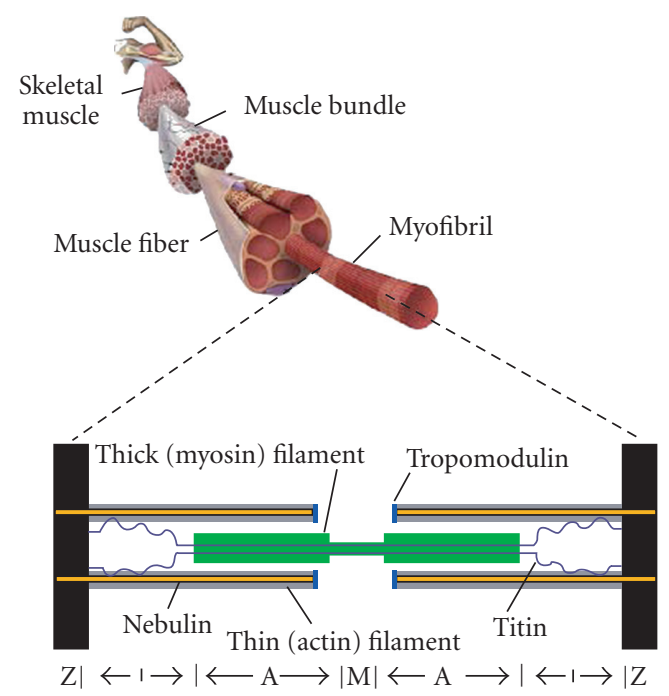

(a)

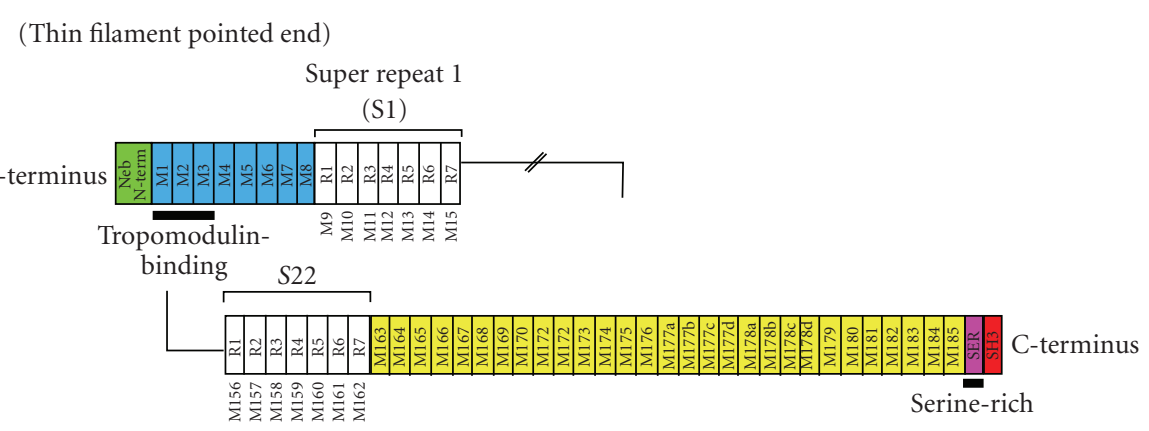

(Thin filament barbed end, Z-disk)

(b)

FIGURE 1: (a) Schematic of the structural organization of skeletal muscle (top) and sarcomere (bottom). (b) Schematic of the human nebulin sequence. Nebulin has a highly modular structure, with, in the central region, (M9-M162) seven modular repeats arranged into twenty-two superrepeats (only two of which are shown).

addition to binding nebulin's $\mathrm{N}$-terminus, binds actin and tropomyosin with high affinity and prevents actin filaments from elongating or shortening at the pointed end [11]. Furthermore, earlier studies revealed that the electrophoretic mobility of nebulin from different muscle types correlates with thin filament length $[5,12]$.

Although the findings discussed above were consistent with the hypothesis that nebulin is involved in specifying thin filament length, direct evidence was lacking. More conclusive evidence for a role for nebulin in specifying thin filament length required studies of muscle that lack nebulin. Such studies were first performed by McElhinny and coworkers [13]. It was found that upon RNAi knockdown of nebulin transcripts in cultured cardiac myocytes, thin filaments elongated to unrestricted lengths, thus supporting a role for nebulin in regulating thin filament length. These experiments were carried out in cardiac myocytes where nebulin expression is less than $1 \%$ of the levels found in skeletal muscle [14]. Thus, to test the role of nebulin in skeletal muscle in vivo, nebulin $\mathrm{KO}$ mouse models were generated $[14,15]$. The first work on these models revealed that in nebulin-deficient skeletal muscle the thin filaments are on average shorter, thus supporting a role for nebulin in the in vivo regulation of thin filament length $[14,15]$. Witt et al. [14] performed an immunoelectronmicroscopy study and reported that thin filament lengths in wildtype tibialis cranialis muscle are a constant $1.2 \mu \mathrm{m}$, but in nebulindeficient muscle are on average $\sim 0.8 \mu \mathrm{m}$, and range from $\sim 0.4$ to $1.2 \mu \mathrm{m}$ (for a schematic, see Figure 2(a)). That such reduction in thin filament length greatly affects force production was illustrated by Ottenheijm et al. [16] by measuring force as a function of sarcomere length for both wildtype and nebulin-deficient muscle. In these experiments, skinned muscle fibers were activated by supramaximal levels of exogenous calcium at various sarcomere lengths and the force response was measured (note that in skinned fiber preparations, factors outside of the myofilaments, e.g., calcium handling by the sarcoplasmic reticulum, do not contribute to force production). As shown in Figure 2(b), the force-sarcomere length relation of wildtype muscle is 


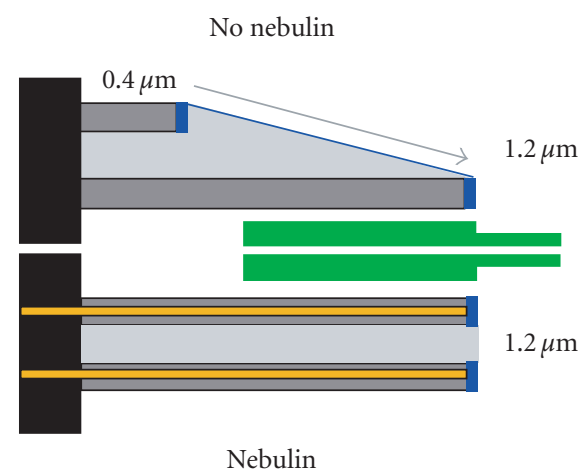

(a)

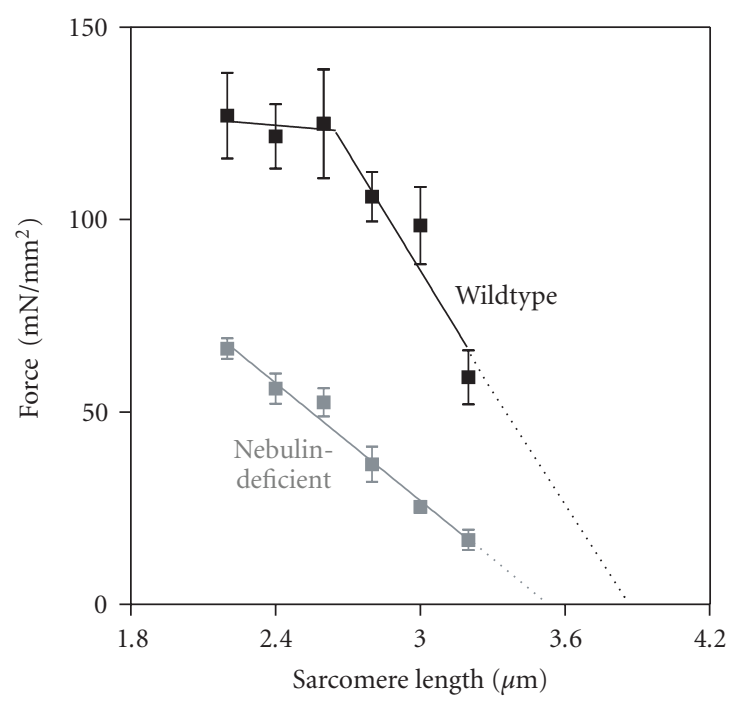

(b)

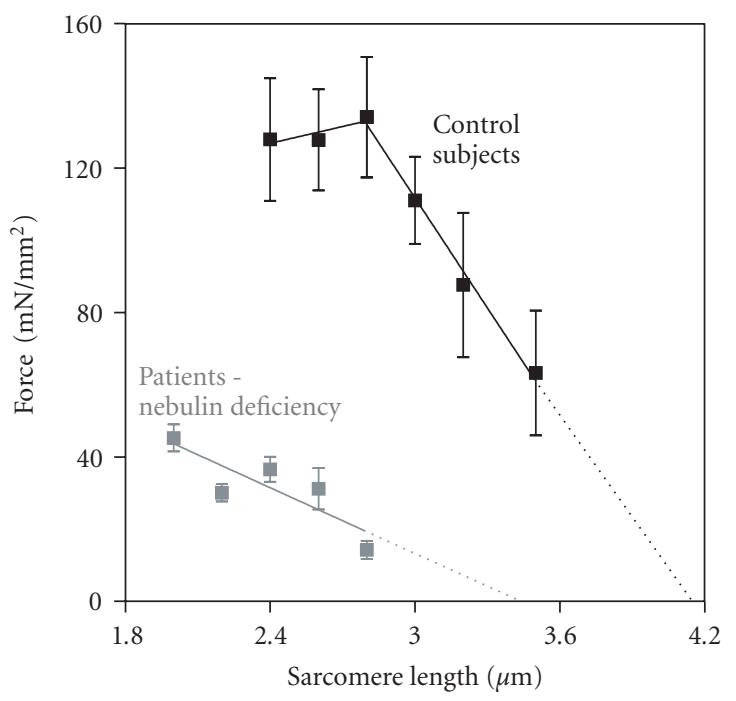

(c)

FIGURE 2: (a) Schematic illustrating the range of thin filament lengths found in nebulin-deficient muscle fibers. Immunoelectron microscopy [14] showed that thin filament lengths in wildtype tibialis cranialis muscle are a constant $1.2 \mu \mathrm{m}$, but in nebulin-deficient muscle are on average $\sim 0.8 \mu \mathrm{m}$ and range from $\sim 0.4$ to $1.2 \mu \mathrm{m}$. (b) The force-sarcomere length relation of murine wt muscle fibers has a characteristic force plateau followed by a descending limb. The force-sarcomere length relation of nebulin-deficient fibers is shifted leftward compared to wt fibers, and the force plateau is absent. (c) Similarly, the force-sarcomere length relation of nebulin-deficient human muscle fibers (dissected from biopsies obtained from patients with nemaline myopathy) is shifted to the left, and the force plateau is absent as well [16].

characterized by a force plateau reflecting optimal thick-thin filament overlap, followed by a descending limb at higher sarcomere lengths reflecting the decreased filament overlap. That the descending limb in wildtype muscle starts at a sarcomere length of $\sim 2.6 \mu \mathrm{m}$ and ends at $\sim 4.0 \mu \mathrm{m}$ suggests a thin filament length of $\sim 1.2 \mu \mathrm{m}$, which is in line with the previously mentioned electron-microscopy data. In nebulindeficient muscle, the shortened thin filaments reduce thinthick filament overlap at a given sarcomere length, impairing force production and resulting in a leftward shift of the force-sarcomere length relation (see Figure 2(b)). Furthermore, when thin filaments are nonuniform in length, no optimal thick-thin filament overlap exists, and consistent with this the force-sarcomere length relation of nebulindeficient muscle lacks the characteristic plateau. Supporting these findings on demembranated muscle, studies on intact nebulin-deficient muscle from another nebulin knockout model [17], in which muscles were activated at various lengths by electrical field stimulation, also revealed a leftward shift of the force-muscle length relation of nebulin-deficient 

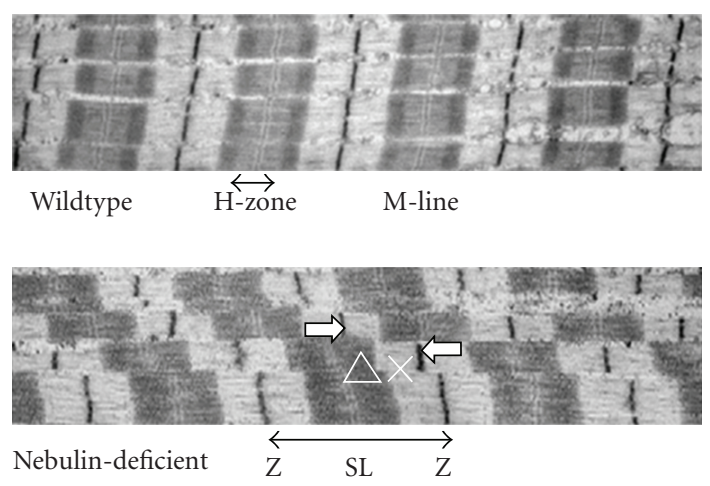

(a)

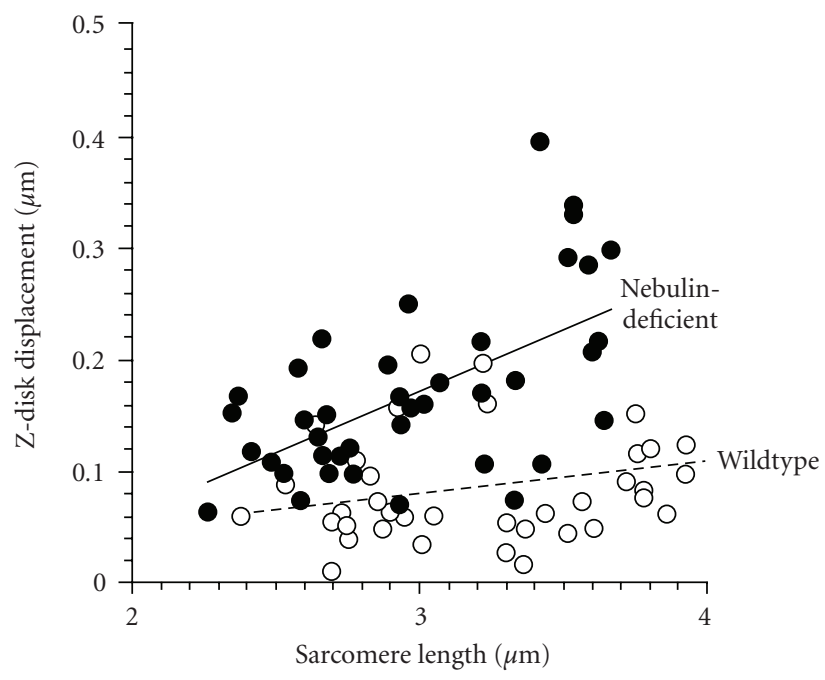

(b)

Figure 3: (a) Wildtype fibers have a regular structure with well-aligned sarcomeres. In nebulin-deficient fibers sarcomeres are misaligned and the Z-disk are out of register. Arrows indicate how Z-disk displacement $(\Delta \mathrm{X})$ was defined. (b) Z-disk displacement is significantly increased in nebulin-deficient fibers. (modified from Tonino et al. [27]).

muscle. Thus, the force-length relation of nebulin-deficient muscle is altered in a manner that is consistent with the presence of shorter thin filament lengths.

Work by Bang et al. [15] on their nebulin KO model, using confocal microscopy on 1-day-old mice, indicated that in the absence of nebulin thin filament lengths are reduced from $\sim 1.15-1.3 \mu \mathrm{m}$ (depending on muscle type) in wildtype muscle to a consistent $\sim 1.0 \mu \mathrm{m}$ in all muscles types. These findings led to the proposal [1] that a nebulinindependent mechanism specifies uniform thin filament lengths of $\sim 1.0 \mu \mathrm{m}$ in all muscle types whereas nebulin is responsible for specifying longer thin filament lengths in a muscle-specific manner. An opposite conclusion was drawn by Castillo et al. [18] who used immunofluorescence microscopy on rabbit muscle and concluded that nebulin specifies the minimum thin filament length $(\sim 1.0 \mu \mathrm{m})$ with a nebulin-independent mechanism regulating the final length according to the requirements of a particular muscle. It is unclear as to what causes this apparent discrepancy between these studies. Detecting differences in thin filament length, especially length gradients, with high precision is challenging and might be hard to accomplish with confocal microscopy, as was done in the two aforementioned studies. In contrast, Witt et al. [14] used electronmicroscopy and decorated thin filaments with gold beads (attached to actin monomers with the actin-binding peptide phalloidin). This made it possible to determine thin filament length gradients and showed that thin filaments varied in length and were on average shorter than in wildtype muscle. Thus, this study does not support the conclusion by Bang et al. [15] that there is a nebulinindependent mechanism that sets a constant thin filament length of $1.0 \mu \mathrm{m}$, but is consistent with the conclusion of Castillo et al. that there is a nebulin-dependent mechanism that sets a minimum thin filament length. To resolve these discrepancies additional studies on a range of mouse muscle types are needed that measure by electronmicroscopy thin filament length and the location of nebulin's N-terminus. It is clear, however, from the above referenced studies that nebulin plays a critical role in regulating thin filament length: in its absence the average thin filament length is shorter and force is greatly reduced.

Nebulin's role in thin filament length regulation provides a mechanism for the first time to explain severe muscle weakness in patients with nemaline myopathy, a debilitating disease frequently caused by nebulin gene mutations and reduced nebulin protein levels $[19,20]$. Indeed, muscle fibers from patients suffering from nebulin-based nemaline myopathy show remarkable phenotypic similarities to fibers from nebulin $\mathrm{KO}$ mice, that is, shorter and non-uniform thin filament lengths and significantly impaired force-generating capacity [16] (see Figure 2(c)). Thus, loss of thin filament length regulation appears to play a prominent role in the pathogenesis of muscle weakness in patients with nemaline myopathy.

\section{Nebulin Maintains Intermyofibrillar Connectivity}

Longitudinally, sarcomeres are connected by Z-disk lattices that anchor thin filaments and transmit force along the myofibril. In the transverse direction, linkage of myofibrils at the Z-disks allows for lateral force transmission and limits the degree to which adjacent myofibrils translocate relative to each other during active contraction or passive stretch, thereby preventing damage to intermyofibrillar membrane systems, such as T-tubules and the sarcoplasmic reticulum. An important protein involved in linking adjacent Z-disks is the intermediate filament protein desmin, which forms a network of filaments that surrounds myofibrils at the level of the Z-disk $[21,22]$. The subunit proteins of desmin filaments 
are elongated coiled-coils with extensive intermolecular ionic and hydrophobic interactions between individual subunits, giving rise to filaments with high tensile strength as well as plasticity [23]. That desmin tethers adjacent Z-disks is supported by work on a desmin $\mathrm{KO}$ mouse in which Z-disk misalignment was shown to occur in stretched muscle [24]. In vitro work, using a yeast two-hybrid approach, suggested that desmin binds to the C-terminal region of nebulin [25], which is anchored in the Z-disk, and recently it was shown that nebulin modules M160-164 are involved in this interaction [26]. These findings lead Bang et al. to speculate that this desmin-nebulin interaction links myofibrillar Zdisks to the intermediate filament system, thereby forming a lateral linkage system which maintains adjacent Z-disks in register. This role for nebulin in intermyofibrillar connectivity was tested recently by studies using a nebulin KO mouse model [27]. In these studies it was found that upon stretch, myofibrils devoid of nebulin translocate to a much higher degree than WT muscle, resulting in much larger Zdisk displacement (Figure 3). Although desmin is present in muscle devoid of nebulin, it is reduced in the intermyofibrillar spaces that surround the Z-disks, suggesting that nebulin is required for proper localization of desmin at the Z-disk. Consistent with this, both knockdown of nebulin with siRNA and overexpression of M160-M170 did not interfere with the formation of normal striation patterns but did prevent desmin localization at the mature Z-disk. Thus, nebulin is required to laterally link myofibrils at the Z-disk by desmin filaments; in the absence of nebulin myofibrillar connectivity is significantly reduced leading to Z-disk displacement.

In addition to linking adjacent myofibrils, evidence also suggests that nebulin's C-terminus regulates Z-disk width. Z-disks of different muscles can vary greatly in width, from less than $100 \mathrm{~nm}$ in fast skeletal muscle to more than $150 \mathrm{~nm}$ in slow skeletal muscle [27]. The importance of regulation of Z-disk width is illustrated by muscle from patients with nemaline myopathy, which displays greatly widened z-disks, including the characteristic nemaline rods [28]. Previously, titin has been suggested to play a role in Z-disk assembly [29]. The Z-disk region of titin contains a family of differentially expressed repeats, the titin Z-repeats [29]. These Zrepeats are a family of $\alpha$-actinin-binding motifs, which are differentially expressed in a tissue- and developmental-stagespecific fashion [29]. As previously pointed out, it is unlikely that the differential expression of the titin Z-repeats alone can determine the Z-disk width, because too few isoforms exist to account for the wide range of different Z-disk widths [30]. Electronmicroscopy on nebulin KO fibers revealed that nebulin-deficient muscle contains electron-dense Z-disk-like bodies that are similar to the nemaline rod bodies that are a hallmark of nemaline myopathy in humans $[14,15,27]$. Furthermore, in nebulin-deficient myofibrils the average Zdisk width is increased by $40-80 \mathrm{~nm}$ depending on muscle type. These findings support a model in which titin, and nebulin together specify Z-disk width, with titin constructing the central region of the Z-disk, including the number and positions of $\alpha$-actinin cross-links and nebulin determining the ending of the Z-disk structure and its transition to the I-band, that is, nebulin functions as a Z-disk terminator. The mechanism by which nebulin terminates the Z-disk might involve interaction between nebulin and Z-disk-localized proteins, such as CapZ. CapZ is a barbed-end actin-capping protein that binds near the C-terminus of nebulin [31]. In muscle fibers devoid of nebulin $[14,31]$ CapZ does not localize properly, allowing the barbed ends of thin filaments to continue to grow beyond the Z-disk resulting in widened Z-disks. Thus, in addition to laterally linking myofibrils at the Z-disk, nebulin regulates Z-disk width. When this feature of nebulin is not present, Z-disks widen, ultimately culminating in the formation of nemaline rods.

In summary, recent studies provide evidence that nebulin's structural roles within the muscle's sarcomere involve (1) specifying thin filament length to optimize thin-thick filament overlap and force production, (2) maintaining intermyofibrillar connectivity, and (3) setting physiological Z-disk widths. These findings provide important novel insights into the role of nebulin in skeletal muscle structure and function and provide new avenues for understanding and combating the severe muscle weakness in nemaline myopathy, a debilitating disease which is frequently caused by nebulin deficiency.

\section{Acknowledgments}

This work was supported by National Institutes of Health, AR-053897 (HG), and by a VENI grant from the Dutch Organization for Scientific Research (CO).

\section{References}

[1] R. S. Littlefield and V. M. Fowler, "Thin filament length regulation in striated muscle sarcomeres: pointed-end dynamics go beyond a nebulin ruler," Seminars in Cell and Developmental Biology, vol. 19, no. 6, pp. 511-519, 2008.

[2] T. J. Burkholder, B. Fingado, S. Baron, and R. L. Lieber, "Relationship between muscle fiber types and sizes and muscle architectural properties in the mouse hindlimb," Journal of Morphology, vol. 221, no. 2, pp. 177-190, 1994.

[3] T. D. Pollard and G. G. Borisy, "Cellular motility driven by assembly and disassembly of actin filaments," Cell, vol. 112, no. 4, pp. 453-465, 2003.

[4] K. Wang and J. Wright, "Architecture of the sarcomere matrix of skeletal muscle: immunoelectron microscopic evidence that suggests a set of parallel inextensible nebulin filaments anchored at the Z line," Journal of Cell Biology, vol. 107, no. 6, pp. 2199-2212, 1988.

[5] S. Labeit, T. Gibson, A. Lakey, et al., "Evidence that nebulin is a protein-ruler in muscle thin filaments," FEBS Letters, vol. 282, no. 2, pp. 313-316, 1991.

[6] S. Labeit and B. Kolmerer, "The complete primary structure of human nebulin and its correlation to muscle structure," Journal of Molecular Biology, vol. 248, no. 2, pp. 308-315, 1995.

[7] A. S. McElhinny, S. T. Kazmierski, S. Labeit, and C. C. Gregorio, "Nebulin: the nebulous, multifunctional giant of striated muscle," Trends in Cardiovascular Medicine, vol. 13, no. 5, pp. 195-201, 2003.

[8] O. Ogut, M. M. Hossain, and J.-P. Jin, "Interactions between nebulin-like motifs and thin filament regulatory proteins," Journal of Biological Chemistry, vol. 278, no. 5, pp. 3089-3097, 2003. 
[9] J.-P. Jin and K. Wang, "Cloning, expression, and protein interaction of human nebulin fragments composed of varying numbers of sequence modules," Journal of Biological Chemistry, vol. 266, no. 31, pp. 21215-21223, 1991.

[10] A. S. McElhinny, B. Kolmerer, V. M. Fowler, S. Labeit, and C. C. Gregorio, "The N-terminal end of nebulin interacts with tropomodulin at the pointed ends of the thin filaments," Journal of Biological Chemistry, vol. 276, no. 1, pp. 583-592, 2001.

[11] C. G. dos Remedios, D. Chhabra, M. Kekic, et al., "Actin binding proteins: regulation of cytoskeletal microfilaments," Physiological Reviews, vol. 83, no. 2, pp. 433-473, 2003.

[12] M. Kruger, J. Wright, and K. Wang, "Nebulin as a length regulator of thin filaments of vertebrate skeletal muscles: correlation of thin filament length, nebulin size, and epitope profile," Journal of Cell Biology, vol. 115, no. 1, pp. 97-107, 1991.

[13] A. S. McElhinny, C. Schwach, M. Valichnac, S. Mount-Patrick, and C. C. Gregorio, "Nebulin regulates the assembly and lengths of the thin filaments in striated muscle," Journal of Cell Biology, vol. 170, no. 6, pp. 947-957, 2005.

[14] C. C. Witt, C. Burkart, D. Labeit, et al., "Nebulin regulates thin filament length, contractility, and Z-disk structure in vivo," EMBO Journal, vol. 25, no. 16, pp. 3843-3855, 2006.

[15] M.-L. Bang, X. Li, R. Littlefield, et al., "Nebulin-deficient mice exhibit shorter thin filament lengths and reduced contractile function in skeletal muscle," Journal of Cell Biology, vol. 173, no. 6, pp. 905-916, 2006.

[16] C. A. C. Ottenheijm, C. C. Witt, G. J. Stienen, S. Labeit, A. H. Beggs, and H. Granzier, "Thin filament length dysregulation contributes to muscle weakness in nemaline myopathy patients with nebulin deficiency," Human Molecular Genetics, vol. 18, no. 13, pp. 2359-2369, 2009.

[17] D. S. Gokhin, M.-L. Bang, J. Zhang, J. Chen, and R. L. Lieber, "Reduced thin filament length in nebulin-knockout skeletal muscle alters isometric contractile properties," American Journal of Physiology, vol. 296, no. 5, pp. C1123-C1132, 2009.

[18] A. Castillo, R. Nowak, K. P. Littlefield, V. M. Fowler, and R. S. Littlefield, "A nebulin ruler does not dictate thin filament lengths," Biophysical Journal, vol. 96, no. 5, pp. 1856-1865, 2009.

[19] K. Pelin, P. Hilpelä, K. Donner, et al., "Mutations in the nebulin gene associated with autosomal recessive nemaline myopathy," Proceedings of the National Academy of Sciences of the United States of America, vol. 96, no. 5, pp. 2305-2310, 1999.

[20] K. Pelin, K. Donner, M. Holmberg, H. Jungbluth, F. Muntoni, and C. Wallgren-Pettersson, "Nebulin mutations in autosomal recessive nemaline myopathy: an update," Neuromuscular Disorders, vol. 12, no. 7-8, pp. 680-686, 2002.

[21] Y. Capetanaki, R. J. Bloch, A. Kouloumenta, M. Mavroidis, and S. Psarras, "Muscle intermediate filaments and their links to membranes and membranous organelles," Experimental Cell Research, vol. 313, no. 10, pp. 2063-2076, 2007.

[22] K. Wang and R. Ramirez Mitchell, "A network of transverse and longitudinal intermediate filaments is associated with sarcomeres of adult vertebrate skeletal muscle," Journal of Cell Biology, vol. 96, no. 2, pp. 562-570, 1983.

[23] M. L. Costa, R. Escaleira, A. Cataldo, F. Oliveira, and C. S. Mermelstein, "Desmin: molecular interactions and putative functions of the muscle intermediate filament protein," Brazilian Journal of Medical and Biological Research, vol. 37, no. 12, pp. 1819-1830, 2004.
[24] S. B. Shah, F.-C. Su, K. Jordan, et al., "Evidence for increased myofibrillar mobility in desmin-null mouse skeletal muscle," Journal of Experimental Biology, vol. 205, no. 3, pp. 321-325, 2002.

[25] M.-L. Bang, C. Gregorio, and S. Labeit, "Molecular dissection of the interaction of desmin with the C-terminal region of nebulin," Journal of Structural Biology, vol. 137, no. 1-2, pp. 119-127, 2002.

[26] G. M. Conover, S. N. Henderson, and C. C. Gregorio, "A myopathy-linked desmin mutation perturbs striated muscle actin filament architecture," Molecular Biology of the Cell, vol. 20, no. 3, pp. 834-845, 2009.

[27] P. Tonino, C. T. Pappas, B. D. Hudson, S. Labeit, C. C. Gregorio, and H. Granzier, "Reduced myofibrillar connectivity and increased Z-disk width in nebulin-deficient skeletal muscle," Journal of Cell Science, vol. 123, no. 3, pp. 384-391, 2010.

[28] C. Wallgren-Pettersson, K. Pelin, K. J. Nowak, et al., "Genotype-phenotype correlations in nemaline myopathy caused by mutations in the genes for nebulin and skeletal muscle alpha-actin," Neuromuscular Disorders, vol. 14, no. 8-9, pp. 461-470, 2004.

[29] M. Gautel, D. Goulding, B. Bullard, K. Weber, and D. O. Furst, "The central Z-disk region of titin is assembled from a novel repeat in variable copy numbers," Journal of Cell Science, vol. 109, part 11, pp. 2747-2754, 1996.

[30] S. Millevoi, K. Trombitas, B. Kolmerer, et al., "Characterization of nebulette and nebulin and emerging concepts of their roles for vertebrate Z-discs," Journal of Molecular Biology, vol. 282, no. 1, pp. 111-123, 1998.

[31] C. T. Pappas, N. Bhattacharya, J. A. Cooper, and C. C. Gregorio, "Nebulin interacts with CapZ and regulates thin filament architecture within the Z-disc," Molecular Biology of the Cell, vol. 19, no. 5, pp. 1837-1847, 2008. 

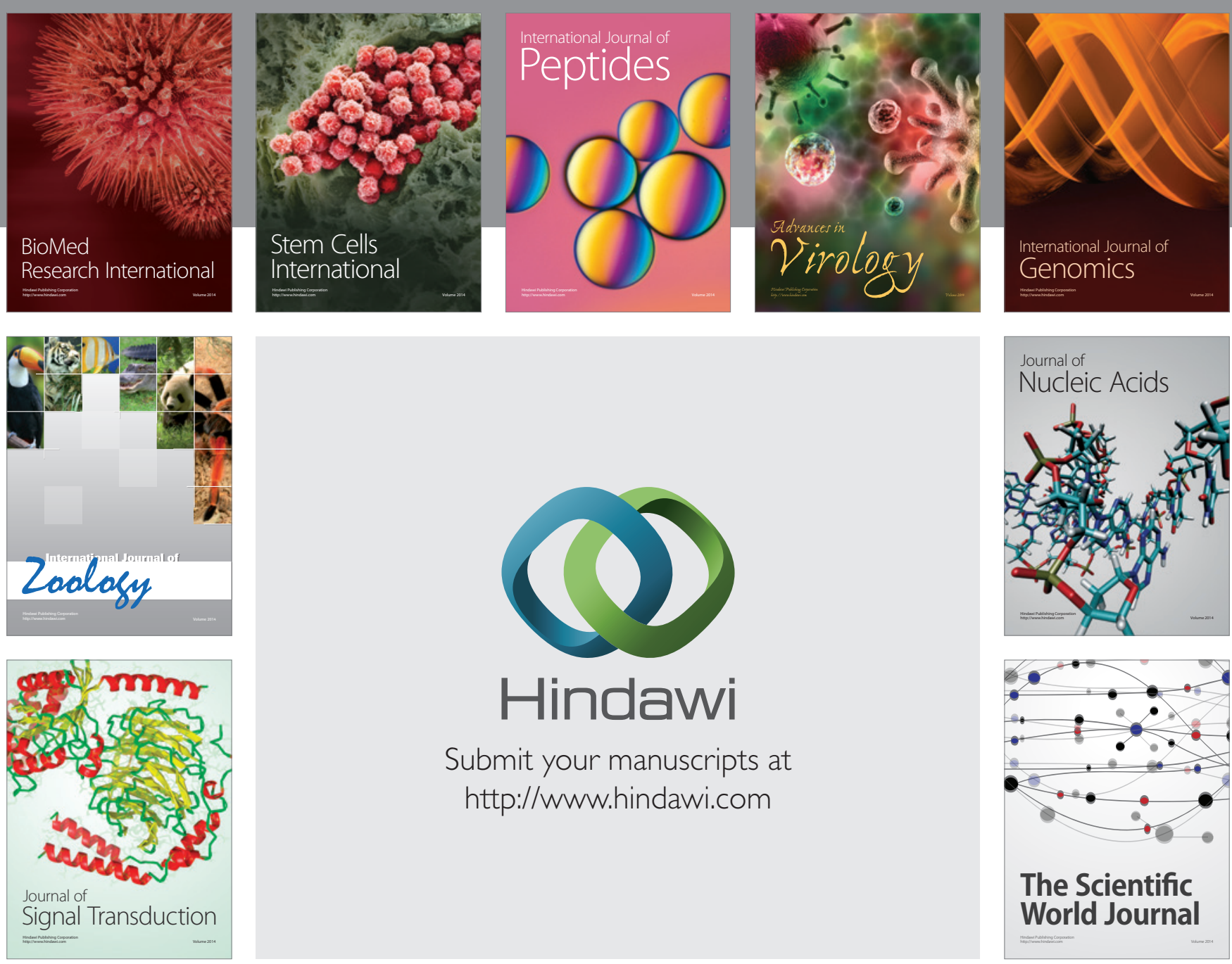

Submit your manuscripts at

http://www.hindawi.com
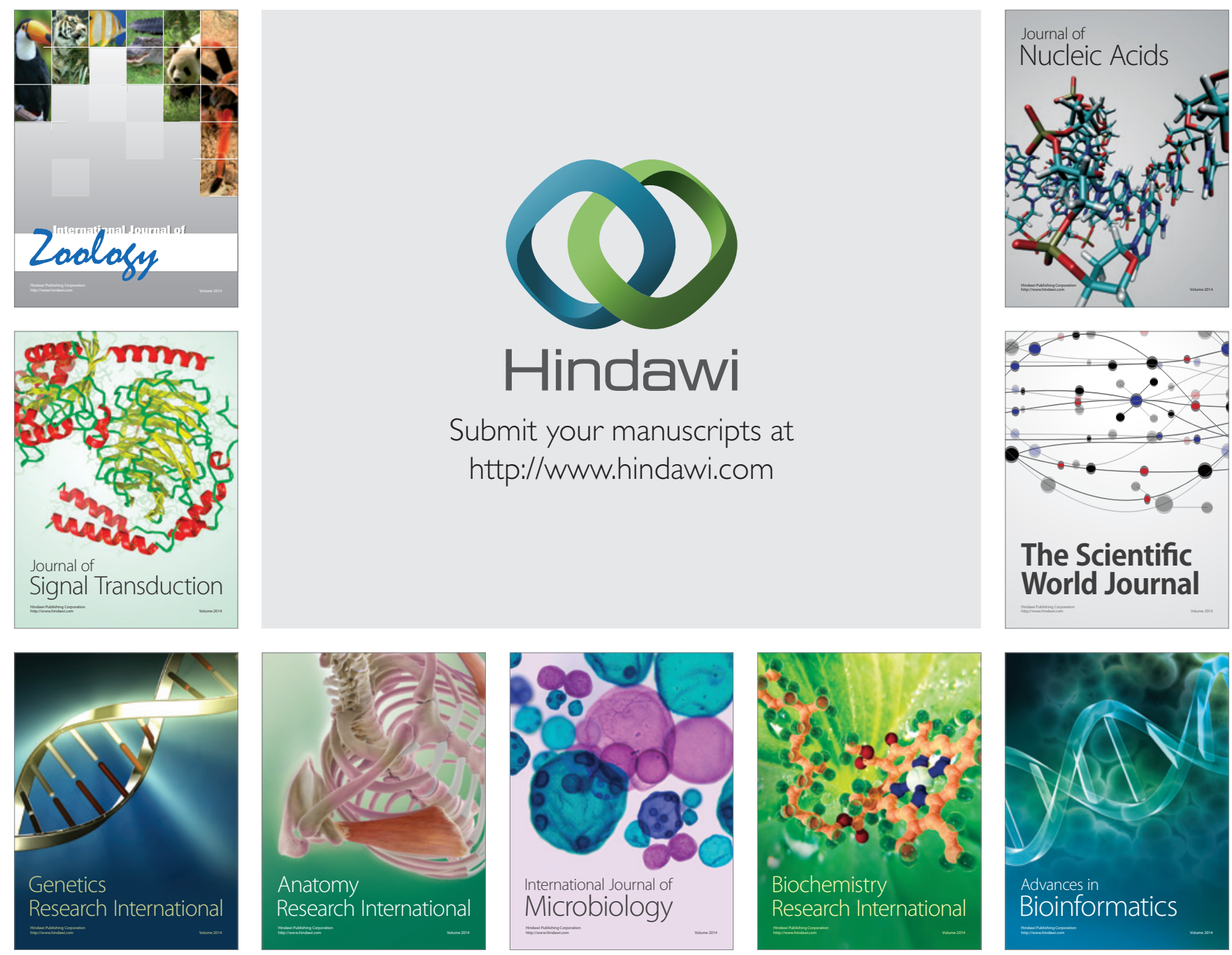

The Scientific World Journal
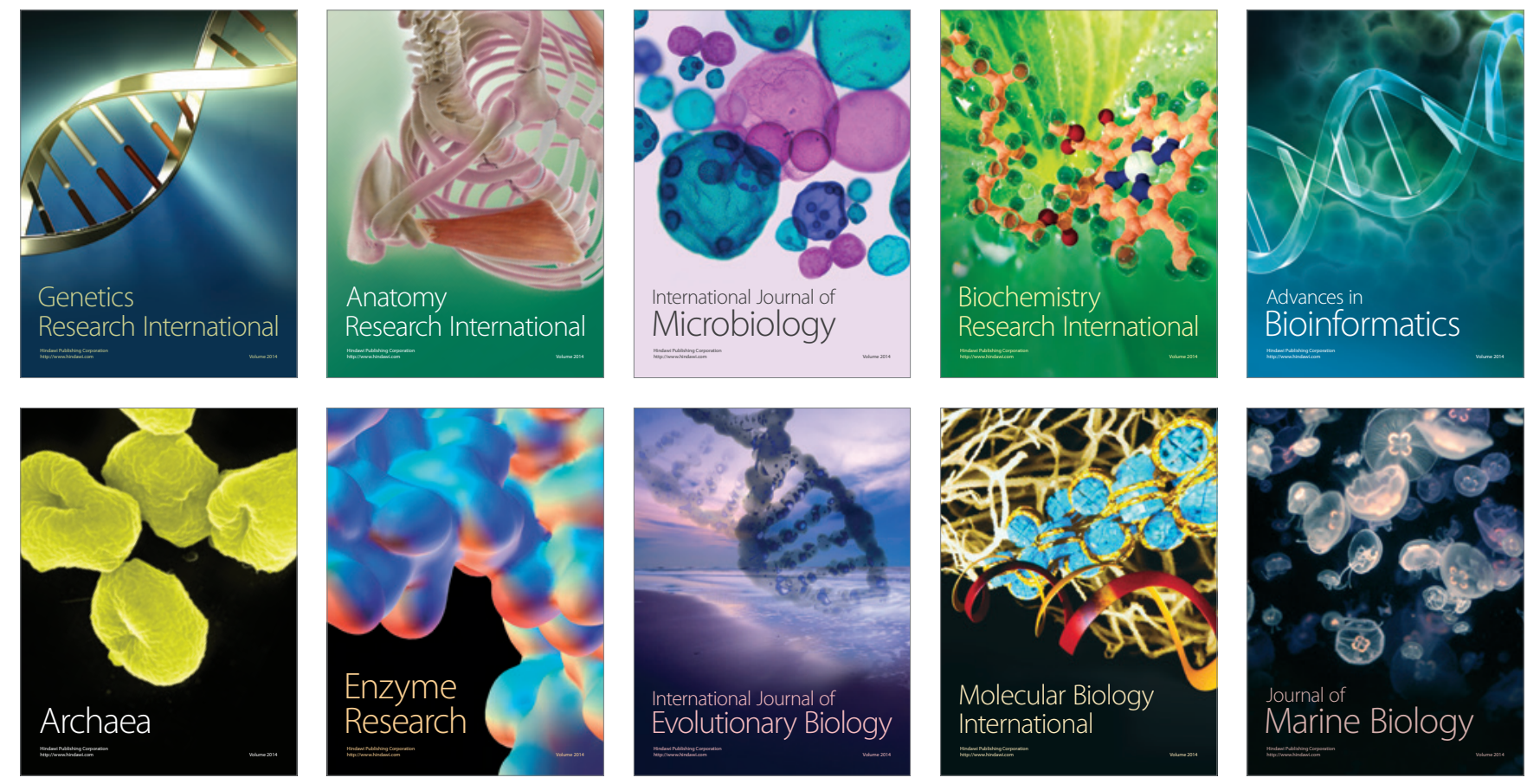